\title{
Das Akustikusneurinom und seine chirurgische Entfernung
}

\author{
Unter spezieller Berücksichtigung der Anforderungen an das pflegerische Personal \\ im HNO-OP
}

\section{Anja Ackermann}

\begin{abstract}
Zusammenfassung
Ein Akustikusneurinom ist ein aus Binde- und Nervengewebe bestehender gutartiger Tumor der von den Schwann'schen Zellen des vestibulären Anteils des 8. Hirnnervs, dem Nervus vestibulocochlearis (Hör- und Gleichgewichtsnerv) ausgeht und im Kleinhirnbrückenwinkel oder im inneren Gehörgang gelegen ist. Symptome sind Hörminderung oder Gleichgewichtsstörungen. Sie entstehen langsam. Bei Hörminderung wird eine Hirnstammaudiometrie (BERA) durchgeführt. Sie stellt die Screening-Methode dar. Das Akustikusneurinom wird mikrochirurgisch therapiert. Man unterscheidet in drei möglichen operativen Zugängen, dem transtemporalen Zugang, dem translabyrinthären Zugang und dem subokzipitalen Zugang. Diese Zugänge wählt man je nach Größe des Akustikusneurinoms. Durch diese drei unterschiedlichen Operationsmethoden und den vielen Instrumenten ist es für uns als OP-Personal eine Operation die eine hohe Anforderung, Geduld, Konzentration und natürlich an erster Stelle die mit großer Sorgfalt und Vorsicht richtige Lagerung des Patienten
\end{abstract}

erfordert. Für das OP-Personal ist diese Operation eine schöne, aber auch durch viele Vorbereitungen eine sehr anstrengende Operation.

\section{Acoustic Neurinoma - The Disease and its Treatment with Specific Emphasis on the ENT - Nursing Staff's Tasks and Duties during Surgery}

Acoustic neurinoma (AN) or vestibular schwannoma is a benign tumour that consists of fibrous and nervous tissue deriving from cells of Schwann's sheath of the vestibular portion of the 8th cranial nerve (vestibulo-cochlear nerve). This tumour is typically located in the inner acoustic meatus, gradually extending towards the cerebellopontine angle. Typical primary symptoms are sudden or slowly progressive hearing loss and vestibular dysfunction (vertigo). Therefore, audiometrical testing with brainstem evoked response audiometry (BERA, ABR) is the first-line screening method in any case of sudden hearing loss and vertigo of unknown origin. Finally, cranial MR imaging is the diagnostic gold standard for the detection of AN. The treat- ment of AN is surgical resection by a microsurgical approach performed by ENT and/or neurosurgeons. There are three surgical options: The translabyrinthine, the transtemporal and the suboccipital (retrosigmoid) approach. The decision as to which one to choose depends on the size of the tumour and the individual symptoms of the patient. Because of the critical, intracranial location, the different surgical methods and the variety of specific instruments, it is a surgical procedure of high complexity - last but not least for the instrumenting staff in the operating room. The necessity for thorough preoperative preparation of the patient, adequate theoretical knowledge of the surgical proceedings and the intraoperative qualified hands-on-assistance set high standards for the degree of training and the skills of the assisting nursing staff. Absolute intraoperative concentration, anticipating perception and subtle instrumenting manners are indispensable demands on the personality of the assisting nursing staff in order to achieve a successful, surgical outcome.

\section{Was ist ein Akustikusneurinom?}

Neurinome der vom zentralen Nervensystem ausgehenden Hirnnerven stellen gutartige Neubildungen (Tumoren) dar, die von den nervenumhüllenden Zellen, den sog. (Peri-)Astrozyten, ihren Ursprung nehmen. Dabei existieren bis heute keine nachweisbaren Ursachen oder Risikofaktoren für deren Entstehung.

OP-JOURNAL 2007; 23: 232-235

(c) Georg Thieme Verlag KG Stuttgart • New York DOI 10.1055/s-2007-989251
Im speziellen Fall des Akustikusneurinoms handelt es sich um einen Nervenscheidentumor der Pars vestibularis als Anteil des 8. Hirnnervs, des sog. Nervus vestibulocochlearis. Daraus leitet sich die medizinisch eigentlich korrektere Bezeichnung Vestibularis-Schwannom für das Akustikusneurinom ab. Die heute landläufig immer noch häufiger benutzte Bezeichnung Akustikusneurinom geht auf die historische Erstbeobachtung der typischen Primärsymptomatik mit Beeinträchtigung des dem Nervus vestibularis direkt benachbarten Hörnerven (Nervus cochlearis) zurück (s. „Typische“ Symptome).

\section{Anatomische Vorbemerkungen}

Zum Verständnis der Lokalisation von Akustikusneurinomen sei - wenn auch sehr verkürzt - die relevante Anatomie des Verlaufs des Nervus vestibulocochlearis (8. Hirnnerv) wiederholt:

Der Nervus vestibulocochlearis verlässt das ZNS im Winkel zwischen Kleinhirn und Pons (Brücke) des Stamm-/Mittelhirns; hieraus resultiert im Übrigen auch eine weitere, synonyme Bezeichnung für das Akustikusneurinom: sog. Kleinhirnbrückenwinkeltumor. Der Nerv zieht dann zusammen mit dem Ge- 
sichtsnerv, dem Nervus facialis (7. Hirnnerv), in einen knöchernen Kanal hinein, den sog. inneren Gehörgang des Felsenbeines. Dieser Gang endet für den Nervus vestibulocochlearis in einem nur Millimeter großen „Raum“ in direkter Nähe zu den Strukturen des Innenohres, bestehend aus Gehörschnecke (Cochlea) und Gleichgewichtsorgan (3 Bogengänge, Sacculus und Utriculus). Dort verteilen sich die entsprechenden, feinen Nervenfasern in Richtung auf ihre Endorgane. Demgegenüber nimmt der Nervus facialis seinen weiteren Weg entlang des Mittelohrraumes und durch den Knochen des Warzenfortsatzes bis in seine einzelnen Endnerven zur Versorgung der mimischen Muskulatur im Gesicht und am Hals.

\section{„Typische“ Symptome}

Die ersten Symptome eines Akustikusneurinoms sind allgemein als eher unspezifisch zu bezeichnen, zieht man die sehr viel häufigeren, gleichartig auftretenden Ereignisse ohne nachweisbares Akustikusneurinom in Betracht (zum Beispiel beim idiopathischen Hörsturz).

Akustikusneurinome manifestieren sich häufig mit einer langsam zunehmenden, einseitigen, typischerweise zunächst die hohen Frequenzen beeinträchtigenden Schwerhörigkeit oder aber auch mit einmalig-fulminanten bzw. rezidivierenden Hörsturzereignissen. Begleitendes oder alleiniges Ohrensausen (sog. Tinnitus auris) ist ein weiteres, mögliches Hörsymptom.

Während die genannten Beeinträchtigungen des Hörsinnes für den betroffenen Patienten nicht selten bleibend und damit allgegenwärtig sind, gehören Gleichgewichtsstörungen, insbesondere Drehschwindel, zwar auch zu den typisch zu nennenden Symptomen bei Vorliegen eines Akustikusneurinoms, allerdings treten diese sog. vestibulären Symptome nur so lange auf, bis zumindest bei einseitigen Tumoren das gleichseitige Gleichgewichtsorgan komplett ausfällt. Nach einer individuell unterschiedlich langen Latenzzeit kommt es dann durch zentral-nervöse Kompensation unter Beteiligung aller Anteile des Gleichgewichtssystems (Groß- und Kleinhirn, Sehsinn, intaktes gegenseitiges Gleichgewichtsorgan, periphere Muskel- und Gelenkrezeptoren) zu einer kompletten Rückbildung der Schwindelbeschwerden („trainierter Schwindelpatient“). Ein lang anhaltender Dau- erschwindel ist daher auch eher als diagnostisches Ausschlusskriterium für das Vorliegen eines (einseitigen) Akustikusneurinoms anzusehen.

Nur bei sehr großen Tumoren oder beidseitig auftretenden Akustikusneurinomen (zum Beispiel im Fall einer Neurofibromatose Typ II) gehören ein Dauerschwindel und Symptome wie Sensibilitätsstörungen im äußeren Gehörgang (sog. Hitselberger-Zeichen), eine periphere Fazialisparese oder Geschmacksstörungen zum typischen Beschwerdebild.

\section{Diagnostik}

Da es sich bei Auftreten erster Symptome in den allermeisten Fällen um Störungen des Hörens handelt, zählen die klassischen, audiologischen Hörtests zu den gängigen diagnostischen Erstuntersuchungen, dabei insbesondere die Reintonaudiometrie. Jedoch erlaubt keines dieser (zumeist rein subjektiven) Verfahren auch nur ansatzweise eine spezifisch für das Vorliegen eines Akustikusneurinoms beweisend wirkende Schlussfolgerung.

Erst die Durchführung einer objektiven, sog. Hirnstammaudiometrie (= BERA = brainstem evoked response audiometry) kann den Verdacht durch Nachweis einer Verzögerung der Nervenleitgeschwindigkeit des betroffenen Hörnerven wahrscheinlich machen. Bei dieser vom Patienten nicht willentlich beeinflussbaren Untersuchung werden den beiden Ohren seitengetrennt jeweils hochfrequente, überschwellige (das heißt sehr laute) Klicktöne zugeführt und das am Hirnstamm ankommende Impulssignal durch am Kopf angebrachte Elektroden abgeleitet. Bei gesunden Patienten kommen die Signale beidseits mit annähernd gleicher Zeitverzögerung an $(\approx 4-5 \mathrm{~ms})$. Bei Patienten mit einseitigem Akustikusneurinom lässt sich demgegenüber eine Verlangsamung der Nervenleitgeschwindigkeit des durch Tumordruck in seiner Funktion beeinträchtigten Hörnerven nachweisen (Seitendifferenz von mindestens $0,3 \mathrm{~ms}$ bei Frauen und von mindestens $0,4 \mathrm{~ms}$ bei Männern). Damit stellt die BERA eine Screening-Methode bei allen unklaren, nicht vom Mittelohr ausgehenden, sog. sensorineuralen Schwerhörigkeiten auf das Vorliegen von Akustikusneurinomen dar.
Der durch eine BERA bekräftigte Verdacht auf eine sog. retrocochleare Läsion, das heißt eine die Nervenleitfunktion des Nervus cochlearis in seinem Verlauf beeinträchtigende Veränderung (zum Beispiel Akustikusneurinom, aber auch Kleinhirnbrückenwinkelmeningeom etc.) kann endgültig nur durch eine Kernspintomografie verifiziert werden. Daher stellt das MRT des Schädels in letzter Konsequenz heute den Goldstandard zur Diagnose eines Akustikusneurinoms dar.

\section{Therapeutisches Vorgehen}

Aufgrund ihrer üblicherweise sehr langsamen Wachstumstendenz ist bei erstdiagnostizierten, kleinen Akustikusneurinomen (Größenausdehnung beschränkt auf den inneren Gehörgang bzw. mit größtenteils im Gehörgang liegendem Tumor, s. „Anatomische Vorbemerkungen") zunächst eine klinische Verlaufsbeobachtung mit regelmäßig im 6-monatigen Abstand durchzuführendem Kontroll-MRT zu erwägen (sog. „Wait-and-scan“-Vorgehen). In diesen Fällen bei ausdrücklichem Therapiewunsch der Patienten sowie grundsätzlich in jedem Fall eines großen Tumors mit dominierender Ausdehnung außerhalb des inneren Gehörganges (Hirnstammkompression!) besteht die Indikation zur operativen Entfernung.

Man unterscheidet generell drei mögliche operative Zugangswege, wobei das chirurgische Vorgehen individuell abhängig gemacht werden muss, einerseits von der Größe des Akustikusneurinoms, andererseits von der noch vorhandenen bzw. fehlenden Hör- und Gleichgewichtsfunktion der betroffenen Seite.

\section{Transtemporaler Zugang}

Diese Operation wird durchgeführt, wenn das noch bestehende Hörvermögen möglichst erhalten bleiben soll und es sich um kleinere Tumoren innerhalb des inneren Gehörganges mit nur minimalem Anteil außerhalb des Gehörganges handelt.

Der Patient wird auf dem Rücken gelagert und der Kopf in eine Mayfield-Dreipunktklemme gefasst. Dieses Drehen darf nicht übertrieben werden, um Durchblutungsstörungen zu vermeiden. Es werden zum intraoperativen Monitoring der Funktion des Nervus facialis (Gesichtsmuskelnerv) und der Funktion des Nervus cochlearis (Hörnerv), Nadel- 
elektroden in die verschiedenen mimischen Gesichtsmuskeln eingestochen bzw. über eine Gehörgangssonde eine Hirnstammaudiometrie (Monitoring BERA) angelegt. Vor Beginn der OP wird oberhalb des Ohres ein $10 \times 10 \mathrm{~cm}$ großes Areal rasiert. Der Patient bekommt nach Absprache mit dem Anästhesisten eine Allgemeinnarkose. Zusätzlich wird der Inzisionsbereich mit einem Lokalanästhetikum infiltriert. Nach sterilem Abdecken des OP-Feldes, Anschließen des Saugers, der bipolaren Pinzette und des Bohrers sowie korrekter Positionierung des OP-Mikroskops durch die OPPflegekraft kann die Operation beginnen.

Die Haut wird in einer bogenförmigen Linie, beginnend kaudal hinter dem Ohr, aufsteigend und nach vorn verlaufend bis in die mittlere Schläfenregion, auf einer Gesamtlänge von etwa $15 \mathrm{~cm}$ inzidiert. Während der folgenden Präparation des subkutanen Gewebes mit Durchtrennung des temporalen Kaumuskels wird ein Muskelstück entnommen, das später zum Verschluss des Defektes des inneren Gehörganges dienen wird. Das Periost der knöchernen Schläfenbeinschuppe wird abgeschoben und ein Sperrer in das Weichgewebe eingesetzt. Mit einem kleinen Rosen-Bohrer werden dann mehrere Bohrlöcher zur anschließenden Entfernung eines Knochendeckels von etwa $2,5 \times 3,5 \mathrm{~cm}$ gesetzt. Der Knochendeckel wird abgehoben und in einer trockenen Schale, bedeckt mit einem feuchten Lappen, bis zur Replantation am Ende der Operation gelagert. Die Dura der mittleren Schädelgrube liegt nun frei. Der nächste Schritt besteht in der Abhebung des Schläfenlappens des Großhirns vom Felsenbeinknochen bei gleichzeitig intakter Durabedeckung des Hirns (extradurales Vorgehen). Somit wird - nach Einsetzen des sog. Fisch-Middle-Forsa-Retractors - ein keilförmiger Operationszugang geschaffen, der in der Folge die von kranial kommende Eröffnung des inneren Gehörganges und die anschließende Entfernung des Akustikusneurinoms aus der Region des inneren Gehörganges ermöglicht. Nach kompletter Tumorentfernung wird das Temporalhirn nach Ausführen des Fisch-Middle-ForsaRetractors schonend zurückverlagert und der Knochendeckel re-implantiert. Zur Vermeidung eines postoperativen Hämatoms wird eine Drainage (ohne Sog!) eingebracht. Zum Schluss erfolgt eine kontinuierliche Muskel- und Faziennaht. Mit der Subkutan- und Haut- naht wird das Wundgebiet verschlossen. Ein zirkulärer, leicht komprimierender Ohrverband beendet die Operation.

\section{Translabyrinthärer Zugang}

Dieser Operationsweg wird gewählt, wenn das Akustikusneurinom nur auf das Innere des inneren Gehörgangs beschränkt ist, sowie gleichzeitig das Hörvermögen erloschen oder so schwer geschädigt ist, dass ein relevantes Sprachverstehen mit dem betroffenen $\mathrm{Ohr}$ nicht mehr gegeben ist. Diese Methode wird speziell auch bei älteren Menschen gewählt.

Es wird ein retroaurikulärer Hautschnitt von ca. $5 \mathrm{~cm}$ Länge angelegt. Nach Freilegung der Warzenfortsatzfläche wird wie bei einer Mastoidektomie - zunächst das Mastoid ausgebohrt. Der Labyrinthblock (= Bogengänge des Gleichgewichtsorgans) wird unter gleichzeitiger Darstellung und Schonung des Nervus facialis exponiert. Anschließend wird der Knochen des Innenohres mit dem Bohrer entfernt. Somit kommt der Fundus des inneren Gehörganges als Ort der Vereinigung der von der Gehörschnecke und dem Gleichgewichtsorgan ausgehenden Nervenfasern zum Vorschein. Von hier wird in der Folge der ausschließlich im inneren Gehörgang liegende Tumor sukzessive freigelegt und abgetragen.

Die Vorbereitungen entsprechen denen zur transtemporalen Entfernung (s. „Transtemporaler Zugang“), wobei der Patient auf das dauerhafte Erlöschen des Hörvermögens auf der operierten Seite hingewiesen werden muss. Auch hier wird das intraoperative Monitoring des Nervus facialis und des Nervus cochlearis angeschlossen, der Kopf des Patienten ebenfalls in eine Mayfield-Dreipunktklemme gefasst. Werden intraoperativ größere Anteile der Dura an der Pyramidenhinterfläche nach Entfernung des Akustikusneurinoms freigelegt, so sollte das ausgebohrte Mastoid bei diesem Zugang mit intraoperativ zu entnehmendem Bauchfett aufgefüllt werden. Hierzu benötigt man Fibrinkleber, der auf die entnommenen Fettgewebsanteile aufgebracht und mit diesen zusammen als Plombe unter leichtem Druck in die Wundhöhle eingelegt wird. Naht und Ohrverband entsprechen im Wesentlichen dem Vorgehen beim transtemporalen Zugang.
Der Vorteil beim translabyrinthären $\mathrm{Zu}$ gang besteht darin, dass keine Hirnanteile verlagert werden und dass der Weg durch den Knochen operiert wird.

\section{Subokzipitaler Zugang}

Diese Operationsmethode gehört der Neurochirurgie. Hierbei handelt es sich um sehr große Tumoren, die im überwiegenden Anteil im Kleinhirnbrückenwinkel liegen.

Der subokzipitale Zugang zur Entfernung eines Akustikusneurinoms bietet seit Einführung der Mikrochirurgie und vielfältiger weiterer Verbesserungen in den letzten Jahren die umfassendste Möglichkeit, Akustikusneurinome jeglicher Größe zu entfernen - bei gleichzeitig guten Chancen, Funktionen zu erhalten. Die Anordnung der Instrumente gleicht der bei den zwei vorgenannten Zugangswegen, jedoch ändert sich die Lagerung des Patienten. Der Patient wird in einer halbsitzenden Position gelagert. Der Oberkörper und die elastisch bandagierten Beine müssen einen Winkel von $30-40^{\circ}$ zur horizontalen Ebene bilden. Nach Anwinkelung der Knie wird der Operationstisch so weit kopfwärts gekippt, dass sich das vorgesehene Operationsfeld auf niedrigstem Niveau befindet - noch unterhalb der Füße des Patienten. Die sorgfältige Polsterung sämtlicher Gelenkregionen vor Beginn der langwierigen Operation ist vom OP-Personal durchzuführen und zum Schutz vor lokalen Nerven- und Gefäßkompressionen unerlässlich. Auch hier wird der Kopf des Patienten mit einer MayfieldDreipunktklemme gefasst, um $30^{\circ}$ anteflektiert und zur Tumorseite rotiert positioniert $\mathrm{zu}$ werden. Zugleich ist ein Mindestabstand zwischen Kinnregion und Sternum von wenigstens $2 \mathrm{~cm}$ einzuhalten. So wird eine Stauchung oder Kompression von Trachea und Vena jugularis vermieden. Durch diese Lagerung ergibt sich für den Chirurgen eine optimale Operationssituation. Darüber hinaus kann in viel größerem Maße auf die bipolare Koagulation kleinerer Tumorgefäße verzichtet und somit für das Nervengewebe schonender operiert werden.

\section{Lagerung beim subokzipitalen Zugang}

Diese Lagerung kann bis zu einer Stunde dauern und bedeutet für das OP-Personal äußerste Konzentration und Ruhe im Operationssaal, um eine korrekte, saubere Arbeit zu leisten (Abb.1). 
Bei diesem Operationszugang wird beim Wundverschluss wieder mit Fibrinkleber gearbeitet. Naht und Verband folgen den anderen beiden Zugängen entsprechend.

Bei allen 3 Operationszugängen werden die Patienten postoperativ für eine Nacht intensiv überwacht. Die Narkose kann zügig ausgeleitet und die Extubation in der Regel sofort oder im Verlauf von 2 Stunden vorgenommen werden. Damit sind sowohl eine gute klinische Beurteilbarkeit in der frühen postoperativen Phase als auch eine rasche Mobilisierung vom ersten postoperativen Tag an gewährleistet. In der Regel ist der Patient nach 8-14 Tagen entlassungsfähig und nach 2-3 Monaten wieder arbeitsfähig. Klinische sowie radiologische Kontrolluntersuchungen sollten nach dem 1., 2. und 5. Jahr erfolgen.

\section{Spezielle Anforderungen an das OP-Personal}

Die Anforderungen an das OP-Personal sind bei allen drei Operationsmethoden sehr hoch. Ein hohes Maß an Disziplin, Durchhaltevermögen, Geduld sowie Fingerspitzengefühl ist notwendig. Die entsprechend qualifizierte OP-Pflegekraft muss darüber hinaus in der Lage sein, die individuellen Bedürfnisse der $\mathrm{Pa}$ tienten unabhängig von ihrer Bewusstseinslage ganzheitlich erfassen und entsprechend fachkompetent handeln zu können. Akustikusneurinomoperationen verlangen spezifisches Fachwissen. Allein die Anzahl der bis zu vier möglichen Instrumentiertische und die speziellen Instrumente, wie zum Beispiel die verschiedenen Wundsperrer, das Rhoton-Micro-Set, die unterschiedlichen Yasargil-Mikro-Federscheren (gerade, $45^{\circ}$ - und $90^{\circ}$-gewinkelt) etc., erfordern ein hohes Maß an Kompetenz und Feingefühl des Personals. Die vielen Instrumente für die genannten Eingriffe setzen also eingehende Kenntnisse und Übersicht voraus. Die sehr feinen, teils filigran gebogenen Instrumente, die in der Handhabung und Pflege besonderer Sorgfalt bedürfen, das Bohrinstrumentarium und das Operationsmikroskop sind allesamt sehr empfindliche, medizinische Geräte und müssen vorsichtig behandelt werden. Das kleine, ohne Mikroskop nicht suffizient übersehbare Operationsfeld, die Geräusche des Saugers und das zeitweilige, konzentrationsbedingte Schweigen des Operateurs verlangen großes Einfühlungsvermögen. Die instrumentierende OP-Pflegekraft muss

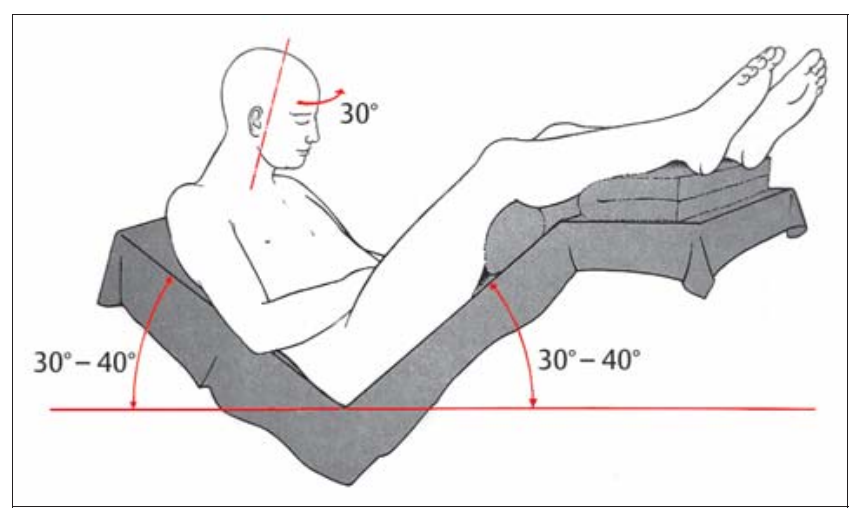

Abb. 1 Lagerung des Patienten beim subokzipitalen Zugang.

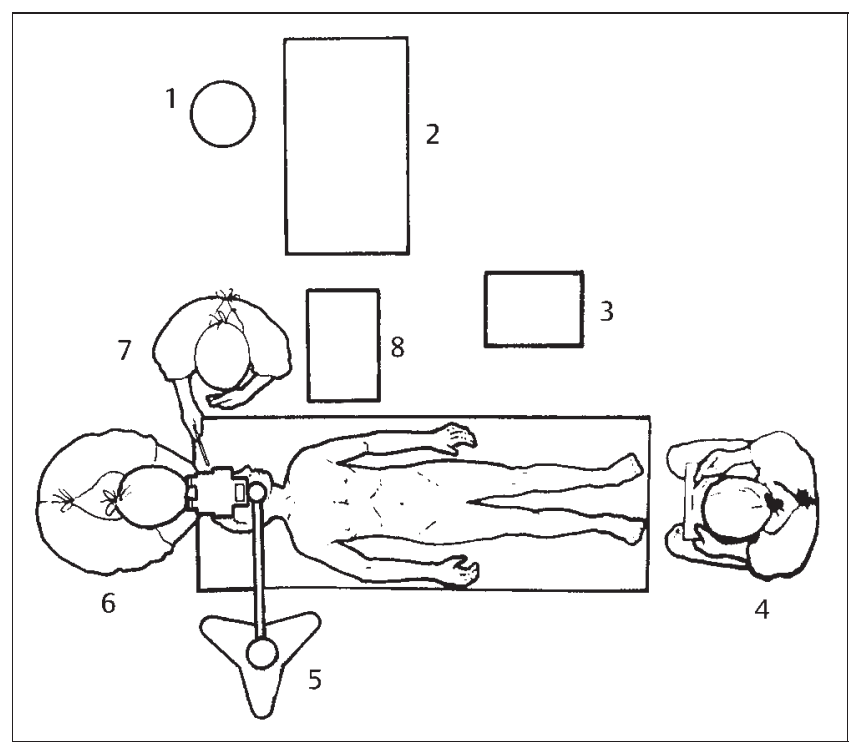

Abb. 2 Eine Akustikusneurinomoperation. (1) Abfall, (2) Instrumententische, (3) Narkosegerät, (4) Anästhesist, (5) Operationsmikroskop, (6) Operateur, (7) instrumentierende Schwester, (8) Instrumententisch.

Geduld beweisen und ruhig arbeiten. Die Lagerung des Patienten muss mit großer Sorgfalt und Vorsicht vonstattengehen, da gerade bei lang andauernd narkotisierten Patienten das Herz-Kreislauf-System, die Atmung, der Stoffwechsel, sowie die Muskelrelaxation ein erhöhtes Risiko für den Patienten darstellen. Deshalb muss sowohl vom Operateur als auch vom OP-Pflegepersonal auf eine korrekte, individuelle Lagerung und sorgfältige Situationsbeobachtung geachtet werden. Da bei dieser Operation auch mit HF-Chirurgie gearbeitet wird, muss der Patient geerdet werden, dabei ist vom Pflegepersonal darauf $\mathrm{zu}$ achten, dass die Elektrode gut am Patienten platziert wird, es zu keinen Druckstellen kommt. Es ist auch im Wesentlichen darauf $\mathrm{zu}$ achten, dass der Patient auf einer trockenen Unterlage liegt, damit bei monopolarer Anwendung keine Kurzschlussströme über den OP-Tisch leiten und es zu Verbrennungen kommen kann. Eine Akustikusneurinomoperation ist für uns als OP-
Pflegekräfte eine schöne, aber auch eine durch die vielen Vorbereitungen anstrengende Operation, die viel Konzentration verlangt (Abb. 2).

\section{Literatur}

${ }^{1}$ Debrand-Passard A, Wunderle G. Pflegeleitfaden OP. Urban und Fischer bei Elsevier, 1996: 498, 576

2 Boenninghaus H-G. Hals-Nasen-Ohrenheilkunde. 9. Aufl. Berlin: Springer, 1993: 158 159

3 Boenninghaus H-G, Lenarz T. HNO. 12. Aufl. Heidelberg: Springer Medizin Verlag, 2004: $119-120$

${ }^{4}$ Helms J, Jahrsdoerfer RA. Kopf- und HalsChirurgie. Band 2: Ohr. Stuttgart: Thieme Verlag, 1996: 285-311

\section{Anja Ackermann}

Abteilungsleitung HNO-OP/Ambulanz

Klinikum Bad Hersfeld

HNO-Ambulanz/OP

Seilerweg 29

36251 Bad Hersfeld 